\title{
Mobility in Collaboration
}

Paul Luff

\author{
WIT Research Group, King's College \\ Campden Hill Road \\ London, W8 7AH, UK \\ +441713334289 \\ Paul.Luff@kcl.ac.uk
}

\author{
Christian Heath \\ WIT Research Group, King's College \\ Campden Hill Road \\ London, W8 7AH, UK \\ +441713334496 \\ Christian.Heath@kcl.ac.uk
}

\begin{abstract}
This paper addresses an issue that has received little attention within $\mathrm{CSCW}$ - the requirements to support mobility within collaboration activities. By examining three quite different settings each with differing technological support, we examine the ways in which mobility is critical to collaborative work. We suggest that taking mobility seriously, may not only contribute our understanding of zurrent support for collaboration, but raise more general issues concerning the requirements for mobile and other technologies.
\end{abstract}

\section{Keywords}

mobile communications, augmented reality, object-centred interaction

\section{INTRODUCTION}

It is a strange irony that given the promise of new spaces and novel environments for collaboration, current technologies appear so rigid. Tools for remote collaboration, such as desktop conferencing, groupware, and more innovative technologies like Clearface [14] and CVEs [3] , are available on devices which are static and tied to the desk. Systems designed for co-present activities (e.g. [16]), such as meetings and decision support, although they are concerned with providing new and innovative environments, are similarly located on inflexible workstations. Even attempts at expanding the workspace through the novel use of multiple or roving cameras have had quite inadvertently severely restrict the movement of individuals both within their own environments and those with which they connected $[5,11]$.

The ways in which mobility features in collaborative work has largely been overlooked within CSCW (but see [2, 20] or exceptions). It has neither formed the focus for developing innovative mobile technologies, nor featured in empirical studies of work and communication. In this paper, we report on studies of communication and collaboration in three very different settings, and discuss the implications of the Jbservations for supporting mobility and more generally enhancing collaborative work. The three settings consist of: primary health consultations; construction sites; and stations on London Underground. In exploring work and

In Proceedings of CSCW'98. November $14-18$, Seattle. pp. 305-314. ACM Pre 1998. communication in these different environments, we consider how individuals rely upon their own mobility, and the mobility of particular artefacts, to support collaboration, and consider resources which might enhance their current tasks and responsibilities.

\section{BACKGROUND}

Within the field of CSCW considerable attention has been paid to the types of technology that could support remote collaboration between individuals. The development of these technologies, often in prototype form, have both informed and been informed by recent debates concerning the possible changing character of organisations and organisational activities. For example, it is widely accepted that we are witnessing the emergence of new forms of organisation, the fragmented or disaggregated organisation which consists of dynamic networks of firms which temporarily coalesce to produce a particular product or service [1]. It is suggested that the emergence of new communication technologies provide the relevant support for these flexible and contingent organisational forms. For example, mobile technologies, whether these are mobile telephones or more sophisticated devices, seem to support such transformations, allowing organisational activities not to be so focused within a particular office or at a particular desk.

In a rather different way, it is also argued that new technologies play an important part in recent attempts to 'reengineer' business processes, to focus on 'core activities' and to facilitate developments such as 'hot desking', where the organisation is less centred on individual members, and more on the activities they have to perform [6]. Systems for collaborative work, would appear to correspond with and facilitate such developments, offering the kind of support for geographically dispersed individuals only previously available within a setting.

For example, some CSCW prototypes provide novel environments in which individual users, or representations of them, can not only have access to distributed colleagues', but can move through, or reconfigure a shared spatial environment $[5,3]$. More conventional systems provide participants with visual and audible access to each other, as well the ability to share and exchange documents and the like. However, despite the contribution of such systems to supporting distributed activities, the technologies are unwieldy and, rather than enhancing an individual flexibility within his or her immediate environment, tie participants to tightly circumscribed regions within their setting. The physical configuration of the technology may appear to be of minor consequence if the system can provide unprecedented access to remote individuals. However, the trend to make particular devices, routinely workstations, increasingly complex, has important consequences for the sorts of activity and collaboration that such technologies can support. By constraining the movement of individuals, and burdening the 
user with a complex, yet inflexible technology, we run the risk of undermining an important resource in collaboration, namely, an individual's ability to reconfigure him or herself with regard to ongoing demands of the activity in which they are engaged. CSCW runs the danger of localising activities, enhancing the facilities available to the individual 'user' whilst undermining flexible and contingent collaboration.

The trend in CSCW to enhance collaboration by providing increasingly complex support on conventional workstations, would appear to run counter to the findings of many workplace studies. Whilst not concerned with mobility per $s e$, it is interesting to note how a range of studies of various settings have demonstrated how participants, as a matter of zoordinating their activities with others, rely upon their own mobility and the mobility of artefacts. For example, our own studies of the control rooms of London Underground, demonstrate how peripheral awareness and participation is dependent upon the ability of particular individuals to ongoingly configure their spatial relationships to enable them to overhear and oversee the contributions of others, whilst rendering their own activities visible. Similarly studies air traffic control, of offices, of news rooms, of ship navigation, of printing processes, of financial institutions, all in various ways reveal how the mobility of personnel and artefacts is zritical to communication and collaboration. And yet many of us, including ourselves (cf. [8, 12, 13]) have been principally concerned to enhancing the shared facilities for individuals on fixed workstations.

By considering, in a little detail, the ways in which personnel in conventional working environments rely upon their own mobility and the mobility of artefacts in accomplishing their work, we may learn a little as to how we might enhance support for collaboration. The settings in question raise rather different issues and pose rather different problems for computer support. In the first case, we reconsider the introduction of computer based records into the medical consultation, and explore how the technology has undermined a critical resource in collaboration; the scological mobility of traditional, paper record. In considering the use of the paper record, we consider how professional medical practice articulates the document to support reading and writing in concert with the real time zontributions of the patient. The construction site raises cather different issues. It provides a brief glimpse of how documents can be manipulated and transported within a domain to support coordination and collaboration. Observation of these indigenous practices, practices which both rely upon and enhance mobility, begin to reveal how system support for mobility can fail, and can fail by virtue of the technology's ability to support the sorts of collaboration afforded by the original document. Finally, we consider London Underground, and a project concerned with supporting mobile station staff. Its aim is to provide supervisors with access to the facilities of a fixed command and control centre wherever they may be around large stations with complex interconnections. By considering how staff coordinate their activities with each other, we can begin to explore the issues that arise in providing multimedia support for mobile collaboration, and in particular in reconfiguring the environment to support awareness, zommunication and cooperation.

\section{MICRO-MOBILITY: MEDICAL CONSULTATIONS}

In the first study we explore the range of ways in which the mobility of a simple paper document is critical to the work and communication of the medical practitioner. The document in question is the medical record in primary health care; a document which is increasingly being enhanced and replaced by computerised records. We note that the paper record supports both synchronous and asynchronous collaboration, and collaboration between both doctors and other professionals, and between the patient and the doctor. In particular we suggest that the medical consultation, like other activities which are largely office bound, points to a form of mobility not normally associated with new technologies, that is 'micro-mobility'; the way in which artefact can by mobilised and manipulated for various purposes around a relatively circumscribed, or 'at hand', domain.

Consider, for example the medical consultation. Over a number of years we have undertaken successive studies of primary health care and shown the various ways in which the record card is critical to professional practice and the interaction between patient and doctor $[7,10]$. The paper medical record consists of a small A5 (7" x 5") envelope containing various pieces of paper and card, including discharge letters, referral notes and test results (see Figure 1).

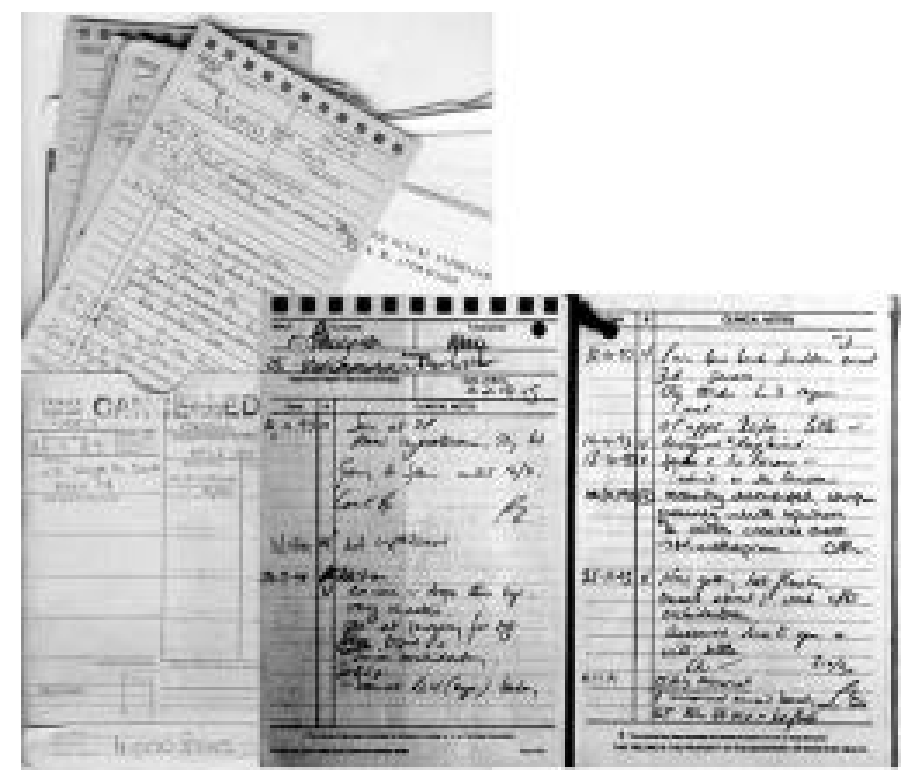

Figure 1: the medical record envelope with typical contents (left), and a detail from a record card (right)

For members in a practice, the paper medical card serves as an important resource for (asynchronous) communication and collaboration. It is transported around the practice or clinic, and even the local region, and some trouble is given to make it available when and wheresoever patient and practitioner meet. Despite the introduction of computerised systems, the paper record remains an important resource in professional medical practice. Its portability is a critical part of its success, as it passes between the hands of different professionals, is carried around the practice, and located in different parts of the office and clinic.

However, it is perhaps in the dealings between patient and doctor, during the consultation, that the mobility of the paper record, becomes critical. For example, the record is read and written in various locations on the desk: it is placed on the knee, held in the hand, and even passed to the patient to read or help decipher the odd entry. It is propped on the desk, whilst the doctor talks to the patient, it can become the focus of gestures and remarks. The ecological flexibility of the record, is a resource in a range of activities, and assists the communicative flexibility of the doctor. For example, whilst talking to the patient, the record can be positioned and manipulated so as to avoid interrupting the free flow of 
the conversation, or on occasions 'foregrounded' so as to give the doctor time to read or enter information. Or consider or example, how a doctor can position the record so as to invite the patient to view the materials; the text itself becoming the focal medium through which the talk is produced and interpreted. The record can even be dismantled, letters removed, or reassembled in different ways; once again the separate mobility of the record's contents exploited for various ways of acting and interacting with the patient.

Unfortunately, this ecological dexterity is not found with zonventional computer systems; indeed, even portables, laptops and the like, are still cumbersome and rigid. It is difficult to position a workstation, both keyboard and monitor in such a way that the doctor can maintain a principal orientation towards the patient whilst reading of entering information, and the physical separation of the area where text is entered from where it is seen, undermines the Joctor's ability to momentarily enter data. The system slutters the desk, and cannot be easily pushed to the rear or brought forward, and whilst monitor can be swung towards the patient, it is difficult to configure a suitable framework around the desk to allow the participants equal access to the information (5). The system is indeed part of the furniture, and as part of the furniture, it demands an orientation from the participants, rather than allowing the participants themselves the ability to ongoingly configure the artefact with regard to the shifting demands of the activity.

The relevance of the micro-mobility of artefacts to the accomplishment of workplace activities is not solely found within medicine or general practice. Indeed, our observations of a broad range of workplace activities in various domains, including news rooms, travel agencies, financial institutions, control rooms, even universities, point to the ways in which the micro-mobility of paper documents is a critical resource in, and feature of, collaboration and interaction. Indeed, with the recent discussions concerning the advantages of paper, the simple yet critical fact that the record is handlable, manipulable, portable, dismantalable and can easily be reordered and reassembled for particular purposes, are critical eatures of the ways in which the document supports the work and interaction between colleagues, and colleagues and their clients. Unfortunately the desk top metaphor, and its influence on the design of digital technologies, fails to take seriously how more traditional artefacts and tools can be reconfigured on the desk with regard to ongoing demands of the work and interactions with others.

\section{REMOTE MOBILITY: A CONSTRUCTION SITE}

In the construction site, we find a more conventional sense of mobility; individuals who move around different physical locations who require access to information and colleagues. In the case at hand, a mobile system was developed and deployed to support the work of foremen, who as a matter of their daily responsibilities, have to document the work of gangs based in different locations around the construction site.

At first glance, the document used by a foreman is not unlike the medical record card. The 'allocation sheet' in its original orm is a paper record of the work which is done by each gang. It records what activities have been undertaken, the time spent, and any problems which have been found and managed (see Figure 2). The record is completed everyday, and is used by a range of personnel to monitor the progress of work, to identify problems, and coordinate activities. The document therefore is both a record of, and a resource in the organisation of work. It is used and referred to by, amongst others, engineers, accountants, surveyors, clerks, and managers.

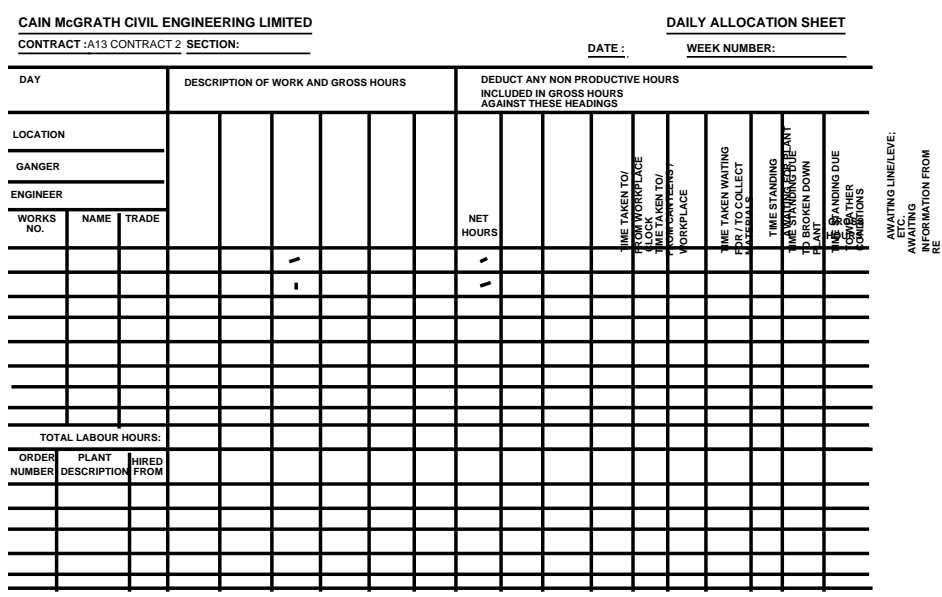

Figure 2: An example allocation sheet, for a gang of steel fixers $(\mathrm{S} / \mathrm{F})$.

Allocation sheets record the work of all the gangs of manual workers out on site and are filled in by those responsible for each gang - the gangers. The completed sheets are then used by a range of personnel within the site hut. Hence, they are a resource for asynchronous and remote communication between those out on site and those in the hut. A foreman, responsible for several gangs typically collects these sheets, checks what has been filled in and then passes them on to the appropriate individuals in the hut. The sheets therefore are mobile; they move around the construction site and are exchanged and assembled within the site hut. Unfortunately, like other paper documents, the traditional allocation sheet is regarded as an outmoded artefact, and it is generally believed that new technologies can provide enhanced support for the documentation, evaluation and management of work on site. These reservations and ambitions informed an exercise undertaken by system developers in the construction company to replace the paper sheets with an electronic system.

In general, it was assumed that a new mobile system would not only fulfil many of the functions currently covered by the paper allocation sheets, but also help document information more quickly and make it more accessible to the various interested personnel. It was also believed that documenting data on a mobile system would allow foremen, where necessary, to discuss various details with gang members and others, wheresoever they might be located. In a sense therefore, it was believed that a mobile system would enhance communication and collaboration, as well as making more bureaucratic activities more efficient. Finally, it was also hoped that the system itself, would provide a resource to foreman to become more proactive; to anticipate problems and difficulties and put solutions into place before trouble emerged.

The allocation sheet is a fairly conventional form that records on what, and for how long, each individual worker has been working. At the end of each day the ganger writes the entries for that day and passes it to the foreman. Once within the site hut the form is the resource for a complex series of activities by various personnel both within the ganger's team and elsewhere in the organisation. So, for example, timekeepers use the allocation sheets for assessing the number of hours worked by particular personnel, engineers and team managers use the sheet for checking on what (and for how long) they have been engaged, and accountants and bonus 
surveyors use them to estimate how much each activity is zurrently costing.

The system designers decided that a Fujitsu 500 Stylistic notepad computer would be an appropriate device to replace the paper allocation sheet. An interface was developed that replicated, as far as possible, the actions accomplished on the paper form. Communication from the device to the site hut was through a mobile phone via a modem.

Needless to say, there were difficulties associated with the introduction of the initial version of the technology. Nevertheless, the system was in continuous use for six months until the team completed work on the construction site. It was then used by other teams for another six months until the completion of the entire construction project. It was used in the everyday work of the project and produced information that was of relevance for the management of the team. For example cost data was produced for the weekly team meeting rather than a week later as with the paper based system.

Despite the success of the system, there were some interesting differences between the aims of the project and the way in which the technology was used. In particular, when it was put into regular service, it was found that it was necessary to employ an additional person whose responsibility was to enter the allocation information and transform it into the form required by other members of staff. This work was not undertaken, as envisaged out on site in zooperation with the gangers, but rather in the site hut itself. In other words, despite the design of system it was neither used as a mobile device nor as a communication tool; rather it remained in a fixed location and was principally used to document data.

This was a consequence of the experiences when the system was tried out in earnest by the foreman during his everyday work around the site.

As mentioned, rather than gangers entering the information or each team, with the introduction of the system these data would be inputted by the foreman for all of the gangs. This would appear to make more efficient use of the technology, particularly as a critical feature of the foreman's work is to roam around the site to monitor problems as they occur. The mobility of the technology could then also provide a resource for discussions between foremen and gangers about problems occurring on the site.

[n some ways, the foreman's mobility around the site is zritical for both his work and is relied on by colleagues. In going around the site, the foreman can monitor the activities in various areas and engage in interactions with participants responsible for them concerning the problems which they are acing. Indeed, these movements appear to be relied on by sther participants; that he will be around at some time, at a particular location or passed when travelling around the site. Such 'serendipitous' contact, or at least the frequent accessibility of the foreman is a critical feature of the his work, both for himself and for others on the site.

However, although the allocation system was mobile and eventually could be used at some speed out on the site, its use tended to transform the ways the foreman worked. Not only did the technology become an explicit topic in the talk between gangers and foreman, but the activity of filling in the allocation sheets became a focus of the work whilst out on site. What had been a brief handover of paper documents, and a transient interaction between ganger and foreman became an extensive activity. Instead of being a resource for talking about the ongoing problems on the site, using the technology actually impeded such discussions. Indeed, in trying to use the system on the site, the foreman would either not be able deal with problems in a particular area or would remain tied to one location. Rather than becoming a resource for mobile collaboration, the technology actually appeared to hinder it.

Following some initial use with the system around the site, the foreman began to use it only in the site hut. He would collect the allocation information from the gangers in a notebook, or even on the old paper allocation sheet and then type all the gangs' information into the system. In this way he could enter the information undisturbed, but this further undermined his routine work of travelling around the site. Rather ironically, the introduction of the mobile system made the user less mobile, less able to monitor the ongoing work and less available to engage in activities with others on site. Hence, after a few weeks it was decided to transform how the technology was to be deployed; another individual would be responsible for entering the information into the system.

These difficulties suggest some misunderstandings by the proponents of the system concerning the nature of the collaborative activities on the site and the technology required to support them. In particular it suggests a misapprehension as to the mobile nature of the work. Because the individuals were mobile it would appear that a mobile technology would be most appropriate. However, the activity it is designed to support does not require the kind of mobility the system provides. Paper allocation sheets are frequently filled out at home, or off site, by gangers. Allowing for the transformation of the activity so actually filling out the sheets can take place around the site requires attention to be paid to how their collaborative production can be supported. In this regard it is unclear whether the technology chosen is the most appropriate one. Indeed, its size, shape and the low intensity of the lighting of the screen made it problematic for items displayed on it to become topics of discussion. It did not even offer the interactional support of the paper allocation sheet.

If this support was considered then attention would have to be paid to the nature of the interactions around the objects and artefacts, the sketches, notes and work schedules, currently utilised within discussions between foremen and gangers. At present it is these that appear to be a resource for collaboration. As in the medical consultation, it may be that a different set of resources are required to support such interactions. Rather than just allowing information to be entered in a range of geographical locations the mobility required may be more in terms of the moment-to-moment accomplishment of the collaborative activities - the micromobility of the activity. It may then be necessary to see how delicate shifts in the accessibility of information, from the individual to the collaborative can be supported during copresent interactions, wherever these may be.

It is also unclear whether the device was appropriate with regard to the more general aim of transforming the activity of the foreman through the use of new technologies. As mentioned, the foreman does have a general concern with the items entered into the allocation sheet, with respect to, for example, retrospectively monitoring the activities which are recorded as having happened. But, his main concerns are with the ongoing occurrences on the site. It is unclear how getting a foreman to fill in the data would help him be more prospective. Indeed, it was curious, that in the initial use of 
the system, rather than transforming the foreman's task to be more proactive and involve more planning and management, the foreman became more involved in bureaucratic activities, keying in data and coding the information against cost categories.

It is not surprising, therefore, that the team decided to continue using the paper sheets and employ someone else to enter and code the information from them. In this way, the oreman could remain mobile around the site, the gangers zontinued entering the information onto the paper sheets and data were available in an electronic form for a range of personnel in the site hut. The use of the computer system, even with the mobile phone connection, remained situated in the hut. Luckily the flexibility of the deployment process, the use of prototyping and the involvement of staff allowed such a transformation in the objectives and design of the system and a technological solution to remain appropriate.

It is a shame that the ambitious aims of the proponents of the system did not quite match the way it was eventually used. But the individuals in the site hut did get the information, by and large, they required in an electronic form and the oreman was not unduly overburdened with an additional and largely irrelevant task. Despite the identification of an individual who needed to be mobile, activities that appeared zumbersome and redundant, and a resource that seemed to require mobile support, the original focus of the exercise appears to have been chosen without paying enough attention to the nature of the activity that the system was intended to replace. This itself did not require mobile support, or at least not of the kind provided; the kind offered on a small. individual system. Even the more radical aim of transforming the work of the foreman, getting him to be involved in planning and more focused discussions about work on the site was hampered rather than supported by the technology.

The development of a mobile technology for foremen may have seemed straightforward. It was focused on individuals who move around a fairly large domain, who need access to remote information and colleagues and it was based on an everyday artefact that was similarly mobile. Nevertheless, the technology, and more specifically the ways in which it could be used, though mobile was not sensitive in the appropriate ways to the practical concerns of those who were meant to use it. Even though critical features relating to mobility were identified, these seemed too crude when the practices of the individuals for which the technology was intended were considered in more detail. To support the mobility required by the foremen appears to require more than transforming a paper document into an electronic one. Serious attention has to be paid to the ways in which personnel interact with colleagues whilst out on site and use various objects and artefacts to accomplish their work with thers.

\section{REMOTE AND LOCAL MOBILITY: LONDON} UNDERGROUND

Unfortunately therefore, the design and deployment of a mobile system to support the work of foremen and others, largely failed to enhance the production or coordination of the various tasks and responsibilities. In part, the failure of the system derived from the ways in which the designers had zonceived of the work of foreman and others, and in particular their disregard for the interactional nature of many of the activities. In a sense, whilst the designers recognised the importance of cooperation, they failed to take into account how the documents themselves could form a basis for realtime collaboration and communication. They also, quite understandably, paid less attention to seemingly latent aspects of particular activities, the way for example foremen's contact with gangs provided a vehicle for more general discussion of site work and its management. In part therefore, the design and deployment of the system misconceived collaboration, and in particular supported asynchronous cooperation whilst disregarding real-time interaction between personnel. In considering mobility, we need to examine the activities in which people engage, with others, when they are 'mobile', and how various tools and artefacts, feature in those activities.

To explore some of these issues, it may be helpful to consider a rather different domain, a domain in which the mobility of staff is critical to the accomplishment and coordination of a range of everyday actions and activities. The case to point is London Underground, and in particular large interchange stations, such as Oxford Circus, Liverpool Street or Waterloo. These stations are very complex 'spaces'; they include platforms at different levels serving different lines, networks of interconnecting passageways (some of which include escalators and lifts), foyers both over and underground, ticket halls and barriers, inter-connecting shopping areas, and multiple entrances and exits. These stations can be dealing with well over a hundred thousand passengers a day, and staff have to oversee these complex ecologies and deal with problems and emergencies when they arise.

The organisational hub of the station is the operations room, commonly known as the 'Ops room'. In the Ops room personnel are provided with radio with which to contact other station staff, a public address system with which to make announcements to passengers, direct lines to Line Control Rooms and the police, switches to open and close gates to the station, and devices to set and reset alarms. Staff are also provided with a bank of CCTV monitors which cover principal areas of the station including the platforms, foyers, escalators and platforms (see Figure 3). The Ops room is normally staffed by one of the duty station supervisors, or a suitably trained station assistant.

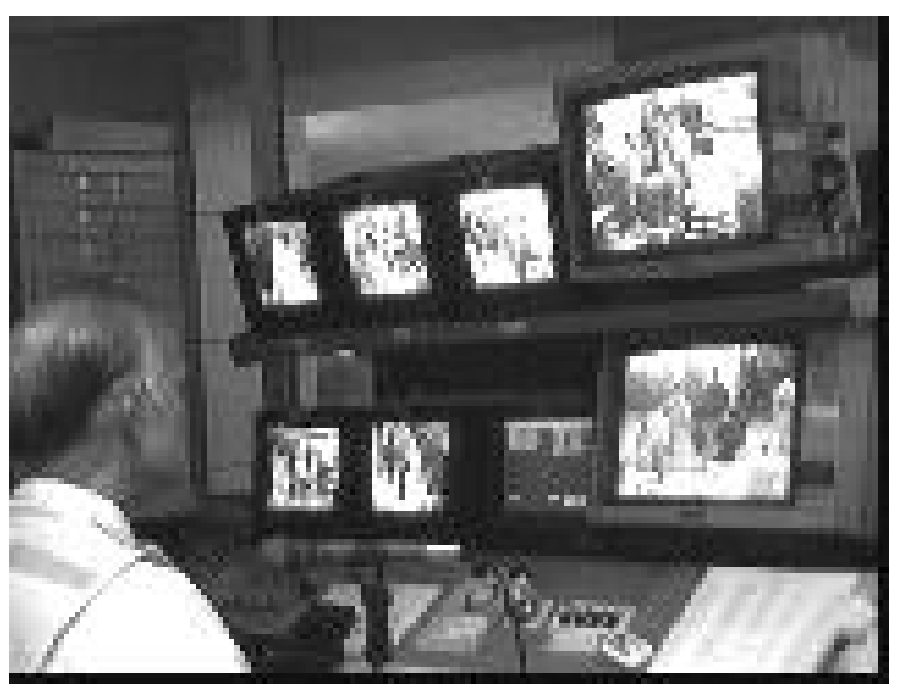

Figure 3: The technology in the Station Ops room

It is widely recognised amongst both staff and management on London Underground, that severe problems derive from the fact that the bulk of information and communication resources are based in one location, namely the Ops room. For example, when a member of staff such as station supervisor leaves the Ops room, he no longer has access to dealing with or overhearing incoming calls from the line control rooms, which provide information concerning 
problems and emergencies, is unable to view areas beyond his/her line of site in the station, or even make announcements to passengers. To have a flavour of the seriousness of this 'resource isolation' of station staff once Jutside the control room, it is worthwhile reading the official report on the fire at King's Cross Station [4] . It is startling to read how station staff, by virtue of their lack of access to ther areas of the station, inadvertently instructed passengers to follow particular routes which were already encased in fire. Moreover, emergency services such as London Fire Brigade also not only had limited knowledge of the station, but also zould not access the detailed paper plans of the domain. More than thirty people died in the incident.

It is perhaps worth briefly describing the sorts of problems which arise in stations and which reveal the necessity for support for mobile staff.

When trains are delayed, especially during peak hours, stations can become severely congested, and because of the increasing number of people on a platform passengers can be pushed towards the edge. Such congestion can require 'station control', where the gates at the entrance of a station are closed to stop more passengers entering. Such a solution requires station staff, preferably a station supervisor, to have access to what is going on in each area of the station and to be able to communicate directly with passengers. Unfortunately, a station supervisor located on the platform has no visual access to what is happening in an entrance hall, and vice versa. He may well have to rely on a colleague's description of the extent and areas of the congestion, and moreover is unable to speak directly to passengers in particular locales. Members of staff require remote access to tomains which will be changing moment-to-moment, access which is currently only available in the Ops room. They also need the ability to discuss and co-ordinate solutions with zolleagues, and to make announcements to passengers in particular locations.

A second example, illustrates how staff in fixed locations, may require variable access to mobile staff and events. A common problem on London Underground, as we have discussed elsewhere, (cf. [8, 9]) are 'suspect packages'. These are packages of any sort, over a certain size, large znough to take a small bomb or incendiary device. It is of zourse, difficult to tell whether a package, such as briefcase or plastic bag, does indeed contain a device, and in general, staff are required to evacuate the station or at least part of the decision. Despite relatively strict guidelines as to when an evacuation should take place, the discovery and identification of a suspect package routinely involves discussion between staff, and sometimes staff, police and specialist bomb disposal teams. In such circumstances various problems arise. Staff in the area of the potential problem are unable to share images of the package and discuss with colleagues based in the Ops room and elsewhere and staff in the area of risk are unable to remain aware of the build up of passengers or other traffic problems. They are also unable to make specific or general announcements to passengers, or have direct contact with the Line Control Room which can make any necessary alterations to traffic flow.

Such information need not just be used in isolation. When zritical incidents occur staff with a range of different roles and responsibilities may not only need access to real-time information but may need to collaborate with others in their immediate vicinity. For example, members of the emergency services need to have information concerning the detailed layout of the station, to see the current status of the incident and its consequences around the domain, and also discuss these with members of London Underground staff at the scene. In less critical circumstances, engineers and maintenance staff not only need visual access to an area where a problem has occurred, but also to discuss possible solutions with station staff. Even in more routine situations staff are frequently faced with having inadequate information when dealing with others in their local vicinity. Station staff whether mobile supervisors or station assistants are continually having to deal with passenger enquiries and yet either do not have the real-time information necessary to answer these or the information given them is available in an inappropriate form. For example. problems and delays on the line, at other stations or on the network as a whole are typically either announced publicly or broadcast to staff through the radios. Hence, there is no (semi-) permanent record to refer to when later these matters seem relevant to particular queries asked by passengers. It would be useful, for example, if station staff could have real-time information that they could at least be used to show to passengers when suggesting the alternatives routes for their journeys or when explaining current problems on the network.

These very brief examples, raise some rather interesting issues with regard to the design of mobile systems. In the first instance, there are a range of facilities that different staff need to access across various locations, including CCTV pictures, information concerning the current state of traffic, audio communications with various personnel, both in and outside the station, and with passengers, gate and alarm operation, and in some cases, access to more general data such as station diagrams, maps of the region and such like. Secondly, different personnel require access to different sorts of information and communication facilities, and in some cases these facilities need to be accessed in different ways. So for example, whilst station assistants need to be able to open and close gates remotely, it may not be necessary for them to be able to make announcements throughout the station, or have general access to CCTV. Perhaps most importantly however, a mobile system needs to support a range of different types of cooperation and collaboration, ranging from simple spoken exchanges through to sharing and discussing images of, say suspect packages, in real time, both with copresent and remote colleagues.

In supporting mobile personnel therefore, a critical consideration is not simply the character of the tasks and responsibilities of the individuals users, but rather how access to such information requires, and engenders cooperation and collaboration. In a sense, supporting station staff on London Underground brings together, requirements for both micro mobility and synchronous collaboration with more conventional considerations for support for asynchronous, cooperation between distributed personnel.

Mobile station staff on the London Underground then both need what might be considered conventional support for mobility - communication and information resources which can be available around the domain - as well as support for micro-mobility - ways of providing and receiving information whilst co-present with others. Examining the requirements in this case may then provide a foundation for a more general consideration of the kinds of mobility which need to be supported in collaborative work.

\section{POTENTIAL TECHNOLOGIES FOR SUPPORTING} REMOTE AND MICRO MOBILITY

In the light of the issues outlined, we are collaborating with London Underground, several radio research and 
development organisations and other transport-related organisations to explore ways of utilising high bandwidth wireless communication systems to support station staff. In particular, the project is examining the ways in which new technologies can provide access to information and communication resources that are sensitive to both the location and circumstances of the users.

Hence, the project is exploring ways of utilising high bandwidth wireless communication systems to extend the zurrent capabilities of station radio, so that it can support the transmission of graphic and textual information about what is happening on the line and the network, access to controls, zommunication and announcement systems and even CCTV images. With such capabilities it is possible to provide a mobile station supervisors with many of the resources available in the Ops room whilst they move around the setting.

In considering the design of the technology however, we have increasingly realised that it may well be a mistake to place access to all necessary resources on the device itself. It is critical for example that the system remains portable, and becomes one of the various tools that station staff carry about with them as part of their normal duties. Moreover, while it may be sensible to provide access to limited textual and diagrammatic information on the device itself, for certain purposes a small CCTV image is of little use.

Consider for example, attempting to discuss the features of a small suspect package on a screen large enough to fit in a pocket, or making a sensible judgement about passenger zongestion from a similarly small display. More importantly, it would be difficult to support even the most basic forms of collaboration, such as discussions concerning timings of trains along the line, let alone a plan of the station in the case of a fire or a video image of a suspect package, on a small mobile devices designed for an individual user. Hence, to provide for more flexible forms of collaboration, allowing for mobility around the domain as well as micromobility around the artefact requires a reconsideration of the kind of support a mobile device can provide.

One approach would be to consider the mobile system as not just the destination of information whilst on the move but also the means through which location-specific information is made available on other devices. Devices such as fixed displays that not only are more suitable for the task-at-hand, but also support co-present collaborative activities. The support for mobile personnel would thus be through a heterogeneous combination of technologies, for example a mix of small mobile devices and larger fixed systems.

So in the case of station supervisors the mobile wireless devices could be used in combination with fixed systems spread around the domain. In some cases this could utilise displays that are currently available, for example at the gatelines of many stations or at the end of most platforms. In thers additional fixed displays could be used which are more ordinarily for other purposes, such as providing passenger information. When in the proximity of such displays it would then be possible for the station supervisors to transform the use of such fixed devices making them active so that information specific to their needs could be displayed.

So, taking a case mentioned earlier, when a 'suspect package' has been reported the mobile device would allow the supervisor to see the status of various alarms, communicate with members of staff both in the station and elsewhere and also make public announcements. Although some access to video would be available on the device, when used in conjunction with a fixed display full CCTV video images could be made available. Such a resource would not only be more suitable to the supervisor's own requirements, but would also provide for shared viewings with other members of London Underground staff and personnel from other organisations. So, for example, a suspect package and the surrounding domain could be viewed with members of the police and other services. Similarly, in the case of a fire, detailed plans of the station could be displayed and used as a resource in discussions with individuals from the emergency services.

Such a configuration can also support other kinds of collaborative activity. In response to a passenger query, for example, relevant network and line information could be brought up on the mobile system and if necessary shifted over to the fixed device. Or, in the case where two members of staff are remotely collaborating over a problem, the fixed display could provide access to a common resource, say, a CCTV image of a particular domain. So when stations are being controlled staff around the gate-line can see what is happening down on the platform and vice versa.

In each of these, the mobile system in combination with fixed devices, provides resources that not only become active and available around the setting, but also support different forms of collaboration. Most importantly, they allow for participants to shift from moment-to-moment, given the circumstances at hand, from the individual and private to the collaborative and public. They provide, to some extent, for micro-mobility within co-present collaborative activities. Moreover, such resources are not tied to any particular location, but available in various locations around a complex setting.

These suggestions currently form the basis for the development of a prototype technology to be used in a trail within London Underground stations. Needless to say, extensive technological testing and design is required to transform this envisionment into a working prototype. The project will have to consider a range of complex design issues, particularly the allocation of functions between fixed and mobile resources, how information can be made readily visible and accessible and how any enhanced communication or collaborative resources relates to existing roles and responsibilities of personnel. Moreover, we will also have to consider ways in which the shift between the private and the public can be facilitated, so that such transformations do not themselves become too intrusive to the demands of the coparticipants co-present in the local environment. The London Underground case provides a useful starting point for considering such issues as the technology may have to be utilised within various quite different kinds of activity and between differing forms of collaboration. For example, the ways in which a resource is made available to a co-located member of staff may differ from how it is distributed to remote participant(s), and these in turn may be different from the ways in which members of staff make public items of information to one or more members of the public. In each case not only the resource but the way it is made available the shift towards the collaborative - needs to be sensitive to the ongoing activities of the participants.

\section{IMPLICATIONS FOR TECHNOLOGIES SUPPORT MOBILITY IN COLLABORATION}

TO

Although the requirements of London Underground staff may appear rather unusual and particular to that setting, this case raises more generic issues with respect to the design and development of new technologies. 
For example, in distributing technologies around the invironment, developing support for station supervisors would appear to be a prototypical case of 'ubiquitous computing' or 'augmented reality' (cf. [18]). However, although typical developments in these areas aim to support tasks and activities by augmenting everyday artefacts with zomputational capabilities it is not all that clear which artefacts are most relevant for such enhancement, or what zapabilities should be augmented. As the case study on the zonstruction site suggests, the apparent ubiquity, mobility or affordances of an artefact may not lead directly to an appropriate device on which to base a design. One way of ocusing the augmentation of artefacts and environments, particularly if such developments are to move outside the laboratory, would be to consider how everyday artefacts can be resources for collaborative activities. This need not just be concerned with augmenting everyday tools and devices with access to remote individuals, Indeed, in the cases zonsidered here, it would appear enough of a challenge to support co-present collaborative activities around a domain. In doing so, it would be important to examine how such technologies might be sensitive to the local situated activities of participants, the ways in which, in real-time, participants delicately shift their orientations to one another, the relation of these to the ongoing talk and the different ways objects are used and moved during an interaction - that is, the micro-mobility of objects.

Such an orientation may also be relevant in settings where participants would not seem to require the use of mobile technologies, say in an office environment like that of the medical consultation. Even here, exploring the micromobility of documents, could suggest ways in which everyday objects, such as the medical record, could be augmented [15]. As in the case of London Underground, supporting the micro-mobility of such a document, may not just require a mobile device, like a PDA or a notepad zomputer, but a combination of these with fixed displays, so that, for example, items and objects could be moved between the individual and private to the collaborative and public. In this case, during a consultation, a doctor could, when appropriate, make items visible to a patient which could become a resource for more focused collaborative activities

Such possibilities are quite distinct from those typically zonsidered in relation to mobile technologies. The potential of mobile devices is usually regarded in terms of their rapacity to receive and transmit information when the individual using them is moving or situated in a range of locations. Even wireless communications aimed at supporting activities in offices are considered in terms the zase by which the mobile devices can be connected to local networks and communicate information (cf. [2, 20]). Though important, such considerations neglect the potential of mobile technologies to support more fine-grain collaborative activities, moving documents, information and objects between devices with different capabilities from moment-tomoment.

Of course, developments such as these require careful zonsideration of a range of conventional issues with respect to the design of the technology. For example, it is important to consider how such capabilities are presented through the user interface. The need to manipulate objects within an interaction across different platforms, places demands on the underlying architecture, particularly with respect to the persistence of objects and how consistency can be maintained between the various devices. Moreover, the heterogeneous use of mobile technologies with fixed devices may require the capabilities, at any time, to be sensitive to the actual location of the device, for example information may need to be displayed on the 'nearest' appropriate fixed display to the mobile device. One of the typical features of system architectures, particularly within CSCW, is that the location of devices, applications and, even users, is transparent. Applications such as those for the London Underground require the architecture to be location-sensitive.

Nevertheless, it appears from the studies considered in this paper that the micro-mobility of objects may be critical when considering how to support co-present, collaborative activities. To provide for this may require not only both mobile and fixed devices, but quite novel support for mobility that focuses on the moment-to-moment manipulation of objects. It may be then that such mundane details as the movement of a document around a desk, the orientations of the participants in a setting, how a document is made visible within the talk of participants in an interaction and how it makes apparent aspects of that talk, could all be relevant for the design of new technologies. These details may not just be of relevance to technologies designed to support similar activities between remote participants, as typically found in CSCW, but for devices that can be used locally when co-participants are co-present. An orientation towards the detailed interactional work which surrounds artefacts may thus bring together contributions from several quite distinct concerns including those of researchers in CSCW, those concerned with innovations in augmented reality and ubiquitous computing and developers of mobile devices and wireless communications.

\section{CONCLUSION}

When we examine workplace activities in a range of settings, not only those considered here, but others including control rooms, financial dealing rooms and architectural practices [12], we see how individuals' orientations towards objects are continually shifting and being transformed with respect to the ongoing interactions and activities of participants. If we take these apparently mundane activities seriously it may be possible to suggest some ways in which they could be relevant for the design of novel technologies.

Each of the studies discussed here have focused on different aspects of mobility within collaborative work. In the medical consultation the use of existing devices, both paper and electronic, suggested some of the ways in which the mobility of an artefact not only supports collaborative activities in and around the wider environment, but also within co-present interaction between doctors and patients. In the construction site, the introduction of a novel mobile technology was examined. This revealed the different ways in which mobility may be relevant for participants in a setting. What may seem an obvious resource to support with a mobile technology may not actually be sensitive to the practical concerns of the participants. In the London Underground observations of the communication and collaboration between staff have revealed a range of ways in which access to information may be relevant to mobile staff. These also suggest some considerations that have to be taken when attempting to provide this information through novel technologies.

These cases, and several related workplace studies, have revealed how the use of objects-in-interaction are delicately interweaved within the interaction and activities of others $[19,12]$ By attending to the resources through which objects are made relevant from moment-to-moment we can see how their mobility is critical in the accomplishment of 
sollaborative activities. Although there have been a few pioneering studies that have explored mobility in the workplace (e.g. [2, 20]). these have tended to focus on tracking individuals who move and roam around a setting. Local mobility in these studies concerns the movements of individuals around a domain and the technological support or them is then considered in terms of providing them with the awareness of others and others of them. This is a quite different kind of local mobility to that which surrounds the ine-grained use of artefacts and provides a real-time resource or moment-to-moment collaboration between participants.

Though technologies have been developed that allow a sertain amount of fluidity between individual and zollaborative activities and between various forms of zollaboration, these means, such as providing different ways of displaying representations or images of participants, have been largely provided on fixed devices. The technology may allow for different views of a document or object and even various types of orientation to a remote participant, but they zonstrain the user within his or her own local domain; the device through which these capabilities are provided usually being cumbersome and unwieldy for use even within its immediate setting. Particularly within interactions, the ways in which a user needs to be mobile have largely been ignored within CSCW.

Developments in augmented reality, at least, focus attention on the object through which computational capabilities are provided. However, such technologies largely ignore how they may be used to support collaborative activities.

Although these oversights might be curious, it may be not so surprising that micro-mobility within collaboration has been ignored by developers of CSCW systems. The local and detailed uses of objects-in-interaction are still relatively unexplored within the social sciences. Indeed, even less subtle ways of individuals, artefacts and activities being mobile within and around a domain have been largely neglected. For example, there has been a tendency for workplace studies, including many of the ones we have been involved in, to focus on 'centres of coordination' [17]. It may be that this focus has led to a tendency to also conceive of technologies to support workplace activities as constrained to particular locales and thence to particular fixed devices. This would be unfortunate, as the very richness of these studies is in their uncovering of the fluid, contingent and situated nature of collaborative work. It may be that we not only need to pursue an examination of the situated use of sbjects and artefacts, but also augment the corpus of workplace studies, so that more open environments are zonsidered.

It may be that in order to consider the requirements for, and development of, more flexible and mobile technologies to support collaborative work. we need to explore in more detail how objects are used in interaction and forms of work where the mobility of participants is critical to that work. Such an examination may not only provide resources for developers of innovative collaborative technologies, but may suggest ways in which conventional personal workstations, mobile devices and systems for ubiquitous computing might be shaped so that they fit the local demands of the participants in the setting. Such analyses may also form the oundation for a more fundamental reconsideration of the uses of artefacts in everyday, social interaction.

\section{ACNOWLEDGEMENTS}

The work reported in this paper is supported by industrial and academic grants including the EC ACTS Programme
Project 'MEMO' (Multimedia Environments for Mobiles) and PORTRAIT (Portable Technologies for Rail Transportation) a DTI/EPSRC LINK Initiative.

\section{REFERENCES}

1. Barnatt, C., CyberBusiness: Mindsets for a wired age. Chichester, Wiley, 1995.

2. Bellotti, V. and Bly, S. Walking Away from the Desktop Computer: Distributed Collaboration and Mobility in a Product Design Team. in Proceedings of CSCW '96. (Cambridge, MA) ACM Press, 209-218, 1996.

3. Benford, S. and Fahlén, L. A Spatial Model of Interaction In Large Visual Environments. in Proceedings of ECSCW'93. (Milan) Kluwer, 109-124, 1993.

4. Fennell, D., Investigation into the King's Cross Underground Fire. Department of Transport, HMSO, (Cm 499) November 1988.

5. Gaver, W.W., Smets, G. and Overbeeke, K. A Virtual Window on media space. in Proceedings of CHI '95 ) ACM Press, 257-264, 1995.

6. Hammer, M. and Champy, J., Reengineering the corporation: A manifesto for business revolution. New York, Harper Collins, 1993.

7. Heath, C.C., Body movement and speech in medical interaction. Cambridge, Cambridge University Press, 1986.

8. Heath, C.C. and Luff, P. Collaborative Activity and Technological Design: Task Coordination in London Underground Control Rooms. in Proceedings of ECSCW'91. (Amsterdam, Netherlands) Kluwer, 65-80, 1991.

9. Heath, C.C. and Luff, P., Convergent Activities: Line Control and Passenger Information on London Underground, in Cognition and Communication at Work, Y. Engeström and D. Middleton (eds.), Cambridge University Press, Cambridge, 96-129. 1996.

10. Heath, C.C. and Luff, P. Documents and Professional Practice: 'bad' organisational reasons for 'good' clinical records. in Proceedings of CSCW '96. (Boston, MA) ACM Press, 354-363, 1996.

11. Heath, C.C., Luff, P. and Sellen, A. Reconsidering the Virtual Workplace: Flexible Support for Collaborative Activity. in Proceedings of ECSCW'95. (Stockholm, Sweden) Kluwer Academic Press, 83-100, 1995.

12. Heath, C.C. and Luff, P.K., Technology in Action. Cambridge, Cambridge University Press, in press.

13. Hindmarsh, J., Fraser, M., Heath, C.C., Benford, S. and Greenhalgh, C. Fragmented Interaction: Establishing mutual orientation in virtual environments. in Proceedings of CSCW'98. (Seattle) ACM Press, 1998 forthcoming.

14. Ishii, H. and Kobayashi, M. Clearface: a seamless medium for sharing drawing and conversation with eye contact. in Proceedings of CHI 92. (Monterey, CA) ACM Press, 525-532, 1992.

15. Luff, P., Heath, C.C. and Greatbatch, D. Tasks-ininteraction: Paper and screen based documentation in collaborative activity. in Proceedings of CSCW '92. (Toronto, Canada) ACM Press, 163-170, 1992. 
16. Stefik, M., Foster, G., Bobrow, D.G., Kahn, K., Lanning, S. and Suchman, L., Beyond the Chalkboard: Computer Support for Collaboration and Problem Solving. Communications of the ACM, 30, 1, 32-47, 1987.

17. Suchman, L., Technologies of Accountability: On Lizards and Aeroplanes, in Technology in Working Order, G. Button (ed.), Routledge, London, 113-126. 1993.

18. Weiser, M., The Computer for the 21st Century. Scientific American, September 1991.

19. Whalen, J., A Technology of Order Production: Computer-Aided Dispatch in Public Safety Communications, in Situated Order: Studies in the Social Organisation of Talk and Embodied Activities, P. ten Have and G. Psathas (eds.), University Press of America, Washington, 187-230. 1995.

20. Whittaker, S., Frohlich, D.M. and Daly-Jones, O. Informal Workplace Communication: What is it like and how might we support it? in Proceedings of CHI ' 94. (Boston, Mass) ACM Press, 1994. 